\title{
ARTICLE
}

\section{Development of high-sensitivity neutron detectors and measurements at the TPS LINAC}

\author{
Yu-Chi Lin ${ }^{\mathrm{a}, \mathrm{b}}$, Kuo-Wei Lee ${ }^{\mathrm{b}, \mathrm{c}}$, Ang-Yu Chen ${ }^{\mathrm{a}}$, Chien-Rong Chen ${ }^{\mathrm{a}}$, Chih-Ching Liu ${ }^{\mathrm{a}}$ and Rong-Jiun Sheu ${ }^{\mathrm{b}, \mathrm{d}^{*}}$ \\ ${ }^{a}$ National Synchrotron Radiation Research Center, 101 Hsin-Ann Road, Hsinchu Science Park, Hsinchu, Taiwan; ${ }^{b}$ Institute of \\ Nuclear Engineering and Science, National Tsing Hua University, Hsinchu, Taiwan; ${ }^{\circ}$ Institute of Nuclear Energy Research, \\ Longtan, Taoyuan, Taiwan; ${ }^{d}$ Department of Engineering and System Science, National Tsing Hua University, Hsinchu, Taiwan
}

\begin{abstract}
Three high-sensitivity neutron detectors have been set up for monitoring neutrons from the commissioning of a 150-MeV LINAC: the first one is a bare cylindrical He-3 proportional counter with a high sensitivity of $\sim 54 \mathrm{cps} / \mathrm{nv}$ to thermal neutrons, the second one uses the same neutron probe but wrapped with $6.5 \mathrm{~cm}$ thick polyethylene as a moderator optimized for neutrons in $\mathrm{MeV}$ range, the third one is an extended version of the second detector with a layer of lead embedded in the moderator aiming to increase the sensitivity of high-energy neutrons. Their response functions to both neutrons and high-energy gamma rays were calculated using MCNPX. Absolute neutron and gamma-ray spectra at the location of measurement were estimated by FLUKA simulations with a high-fidelity geometry model. Detector responses obtained by folding the spectra with response functions were analyzed and compared with measurements. This paper presents the design and characteristics of these detectors as well as a data analysis of the measured results taken during a full-power LINAC operation.
\end{abstract}

Keywords: neutron detectors; high-sensitivity; Monte Carlo; response functions; LINAC

\section{Introduction}

National Synchrotron Radiation Research Center (NSRRC) in Taiwan is now constructing its second light source facility called Taiwan Photon Source (TPS) to meet the increasing demand for brighter synchrotron $\mathrm{X}$-ray source. The accelerator system consists of a 3.0-GeV electron storage ring with a circumference of $518.4 \mathrm{~m}$, a concentric booster synchrotron with the circumference of $496.8 \mathrm{~m}$, and a pre-injector of $150-\mathrm{MeV}$ electron LINAC [1]. Due to some delay of the TPS civil construction, NSRRC has prepared a simple rectangular shielding room (Figure 1) made of $1 \mathrm{~m}$ thick concrete for the assembly and acceptance test of the outsourcing LINAC.

The TPS LINAC is essentially concatenated by three consecutive sections of linear accelerators with a nominal output of $2.25 \mathrm{~W}(150 \mathrm{MeV}, 5 \mathrm{nC}, 3 \mathrm{~Hz})$ electron beam. A $15 \mathrm{~m}$ long transfer line is used to guide the accelerated electrons from the LINAC outlet to the beam dump. The transfer line consists of four quadruples and a dipole magnet arranging in an order of Q1, Q2, Q3, $\mathrm{D} 1$, and Q4 along the beam trajectory. The dipole D1 was designed to deflect the electron beam horizontally by 10-degree for diagnosis purpose. A dedicated beam dump made of iron core surrounded with thick lead and polyethylene was installed at the end of the transfer line to stop the beam. To ensure radiation safety of personnel

*Corresponding author. Email: rjsheu@mx.nthu.edu.tw around the area, gamma-ray and neutron dose rates outside the LINAC room were repeatedly measured and reviewed during the commissioning. The location of the LINAC room is not far from a densely populated building and therefore the dose rates outside the shielding were asked to keep as low as possible. To be able to clearly identify radiation changes above background levels and perform reliable dose rate measurements, high-sensitivity radiation detectors are necessary.

\section{Materials and methods}

\subsection{Neutron detectors}

The electron beam dump has been designed to contain a full-power electromagnetic shower and to attenuate the resulting secondary gamma rays and neutrons to some extent. If all the accelerated electrons were perfectly dumped, dose rates outside the shielding room should be as low as comparable to the natural background. For monitoring gamma rays from the LINAC operation, we used a High Pressure Ionization Chamber (HPIC) model RSS-131-ER from GE Reuter-Stokes, which is designed to measure low-level gamma-ray radiation and capable of quickly identifying small increases above background level. However, commercial neutron area monitors usually do not have enough sensitivity to detect neutron dose rates of natural background. Searching for 


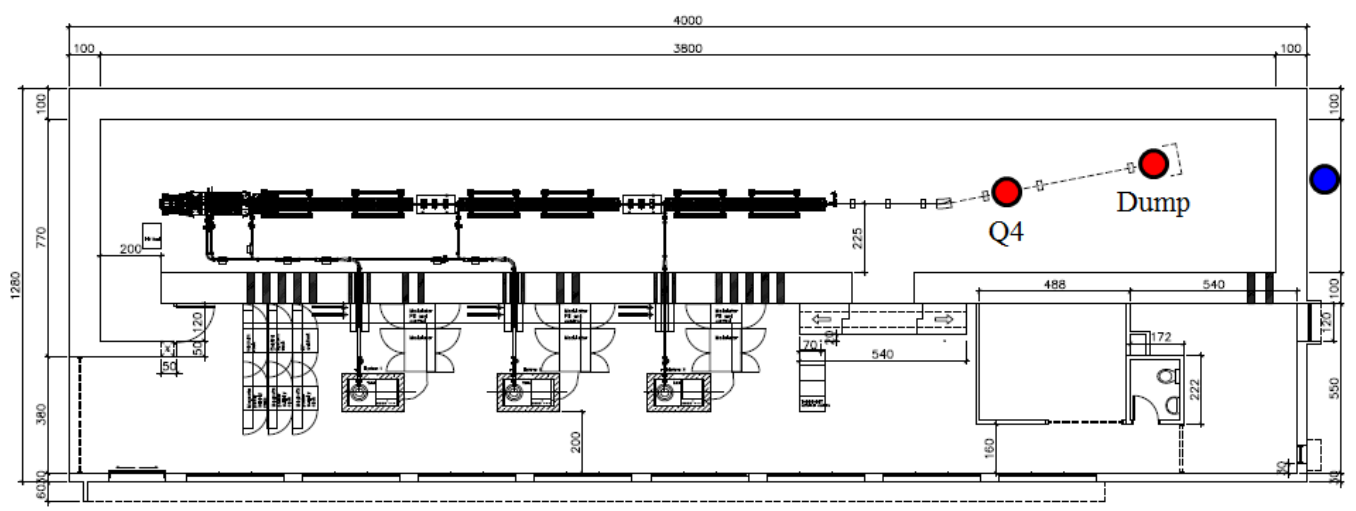

Figure 1. Layout of the LINAC room showing two possible beam loss points at Q4 and the beam dump (red spots), as well as the location of measurement (blue spot).

high-sensitivity neutron detectors, we decided to use self-assembled moderated-type neutron detectors with high-efficiency neutron probes in the center.

He-3 proportional counter model RS-P4-0818-202 from GE Reuter-Stokes was selected as the central neutron probe. It is a cylindrical tube with an active volume of $2.54 \mathrm{~cm}$ in diameter and $45.72 \mathrm{~cm}$ in length and filled with $4 \mathrm{~atm}$ pure helium-3. Its sensitivity to thermal neutrons is about 54 counts per second per unit neutron flux (cps/nv). Three different detector configurations were assembled for monitoring neutrons from the LINAC operation. The first one is just the bare He-3 proportional counter; the second one uses the same type of neutron probe in the center and surrounded by 6.5 $\mathrm{cm}$ thick polyethylene to increase the detection efficiency for fast neutrons and to maximize the neutron counting for natural background; the third one is basically the same as the second detector, except that a layer of cylindrical polyethylene from the radius of 3.3 to the radius of $3.8 \mathrm{~cm}$ is replaced by lead to increase its sensitivity to high-energy neutrons. The three neutron detectors are denoted respectively as "Bare", "PE", and " $\mathrm{PE}+\mathrm{Pb}$ " in the following sections. Figure 2 shows a photo of these three self-assembled high-sensitivity neutron detectors and a HPIC for measuring gamma-ray dose rates.

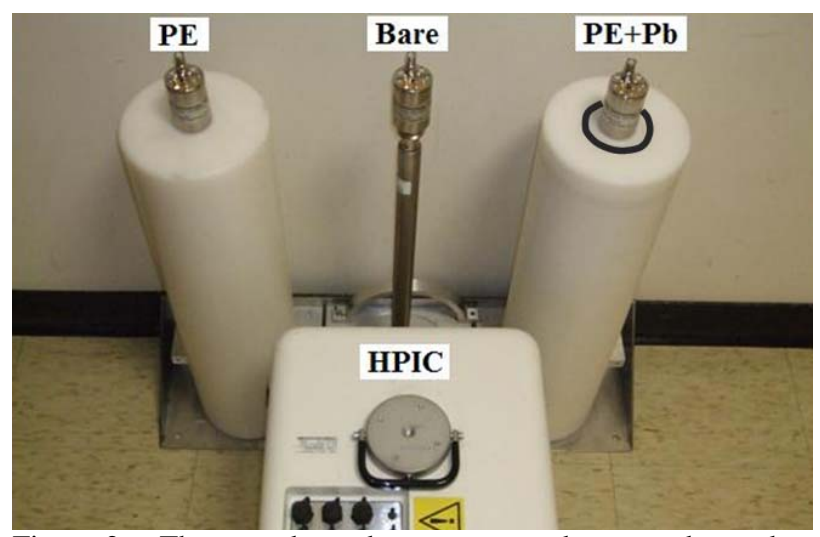

Figure 2. Three moderated-type neutron detectors denoted as Bare, $\mathrm{PE}$, and $\mathrm{PE}+\mathrm{Pb}$, and a high-pressure ion chamber (HPIC) for gamma-ray dose rate measurements.

\subsection{Response functions and radiation field calculations}

Knowing the response function of a detector is essential for a meaning application in radiation measurement. For the three self-assembled neutron detectors, we utilized MCNPX [2] with the LA150 neutron cross-section library to calculate their response functions because it has continuous-energy neutron cross sections up to $150 \mathrm{MeV}$. Above that, MCNPX uses the embedded nuclear models to predict the interaction probabilities of neutrons with matter instead of using evaluated nuclear data. A detector response function was estimated by simulating a broad parallel beam of monoenergetic neutrons or gamma rays incident on the detector surface and scoring the number of ${ }^{3} \mathrm{He}(n, p){ }^{3} \mathrm{H}$ reactions occurred in the detector active volume. Full details of the neutron probe and moderator design were included in the calculation model. The integral response function of the PE detector was tested and verified in a standard neutron calibration room.

In addition to detector response functions, we also need absolute neutron and gamma-ray spectra at the location of measurement in order to estimate the detector responses such as counting rates or dose rates, which can then be compared with measurements. FLUKA $[3,4]$ was used to simulate the radiation field around the LINAC area. It is a three-dimensional multi-particle Monte Carlo transport code capable of simulating hadronic and electromagnetic interactions, charged particle tracking, low-energy neutron transport and full tracing of secondaries. As illustrated in Figure 1, a rather detailed geometry model including main components of the accelerator system, the structure of shielding room and local shielding arrangement was built aiming to predict a reliable radiation environment around the area during the LINAC operation.

\section{Results and discussion}

\subsection{Response functions of neutron detectors}

Figure 3 shows the MCNPX-calculated responses of three self-assembled neutron detectors (Bare, PE, and 
$\mathrm{PE}+\mathrm{Pb}$ ) to neutrons with various energies. Apparently, the bare detector is only sensitive to thermal neutrons. Surrounding by a $6.5 \mathrm{~cm}$ thick polyethylene as the neutron moderator, the PE detector is optimized for detecting neutrons in $\mathrm{MeV}$ range. The $\mathrm{PE}+\mathrm{Pb}$ detector is an extended version of the PE detector with a layer of lead embedded in the moderator, which is intended to increase the sensitivity of high-energy neutrons $(\geq 10$ $\mathrm{MeV})$ through $(\mathrm{n}, 2 \mathrm{n})$ reactions in lead. To validate our MCNPX calculation model, we have simulated an irradiation experiment of the PE detector in a standard neutron calibration room using a well-defined Cf-252 source. The calculated counting rate agrees well with the measurement; their difference is less than $2.5 \%$. Also, the ambient dose equivalent conversion factor of the detector was determined to be $0.0323 \mu \mathrm{Sv} \mathrm{h}^{-1}$ per cps.

Not only neutrons but also high-energy photons could cause counts to be registered by the neutron detectors. Bremsstrahlung photons, which usually dominate the radiation field in an electron accelerator, have a continuous energy distribution up to the primary electron energy. High-energy gamma rays may contribute to a neutron detector through an emission of photoneutrons, especially for a detector with high-Z materials in its composition. The threshold energies of dominant photonuclear reactions are about 5-10 MeV for high-Z materials and the thresholds become much higher for low-Z materials [5]. Responses of these three neutron detectors to high-energy gamma rays were evaluated using MCNPX and the results are shown in Figure 4. Starting from 5-8 $\mathrm{MeV}$, gamma rays contribute to the detector responses to some extent, especially for the extended neutron detector $(\mathrm{PE}+\mathrm{Pb})$ due to the relatively low threshold and high photonuclear cross sections of lead. Although the absolute magnitude of this gamma-ray response function is much lower than those neutron response functions, it is possible to have a non-negligible contribution to the detector if there were intense high-energy gamma rays in the area of interest.

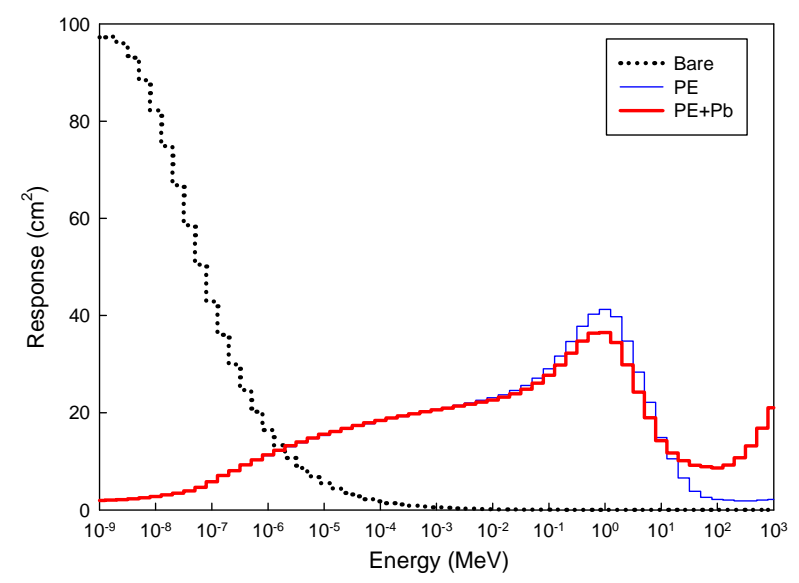

Figure 3. Response functions of three neutron detectors (denoted as Bare, $\mathrm{PE}, \mathrm{PE}+\mathrm{Pb}$ ) to neutrons with various energies.

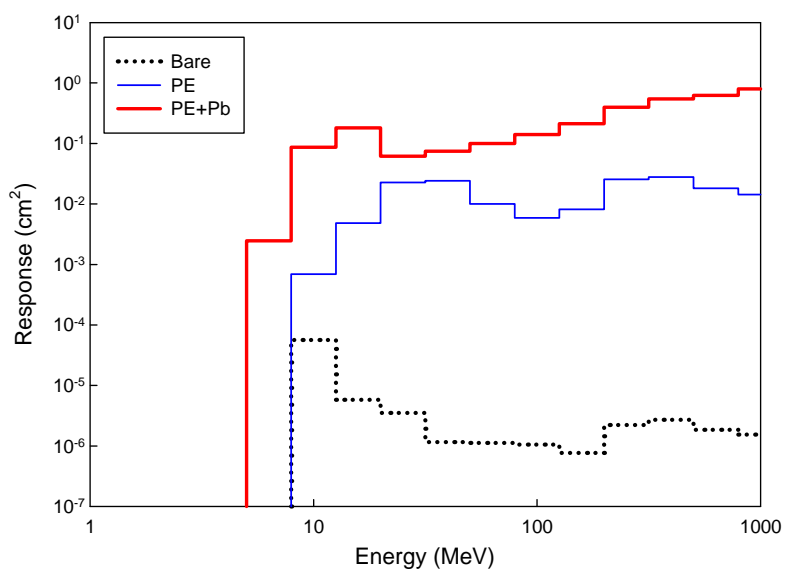

Figure 4. Response functions of three neutron detectors (denoted as Bare, $\mathrm{PE}, \mathrm{PE}+\mathrm{Pb}$ ) to gamma rays with various energies.

\subsection{Radiation field at the TPS LINAC}

When a beam of $150-\mathrm{MeV}$ electrons strikes a material, it will cause an electromagnetic cascade. Secondary gamma rays are the main component of prompt radiation field outside the shielding. Neutrons from photonuclear reactions are outnumbered by orders of magnitude by gamma rays. However, neutrons are substantially more penetrating and may constitute appreciable radiation dose behind a thick shielding. Here we are interested in details of the radiation field outside the downstream shielding wall, where the dose rates are usually the highest around the area. Three neutron detectors were tested at the location. Realistic beam loss assumption is the key for performing an accurate radiation field simulation. According to beam diagnosis information and our previous study on beam loss scenarios for the LINAC operation [6], there are two most probable beam loss locations: one is the design destination of all electrons, i.e. the beam dump, and the other is at the transfer line near the last quadruple Q4. Figures 5 and 6 show the resultant neutron and gamma-ray spectra scored at the measurement location for assuming $100 \%$ beam lost at the two locations, respectively.

A comparison of neutron and gamma-ray spectra in Figure 5 or 6 reveals several important characteristics of the radiation field. First, the neutron flux is much lower than that of gamma rays in both cases, in particular for the second scenario assuming beam lost at Q4. Note that a multiplication factor of 1000 is applied in Figure 6 for the neutron spectrum to fit in the same scale. In addition, the location of beam loss affects significantly the overall intensities and energy distributions of neutrons and gamma rays at the location of interest. Although more than $95 \%$ of the accelerated electrons are actually lost at the beam dump, these electrons are not important in terms of dose rates outside the shielding because of a conservative design of beam dump. In contrast, a small portion of the beam lost along the transfer line may cause significant gamma-ray or neutron dose rates at the downstream. As to the neutron and gamma-ray spectra at the measurement location, the energies of most gamma 


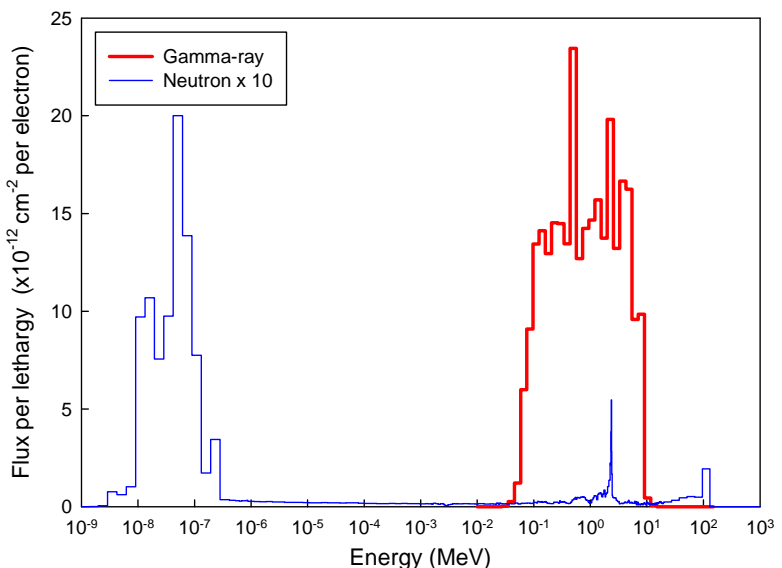

Figure 5. Neutron and gamma-ray energy spectra at the measurement location calculated for electrons lost at the beam dump.

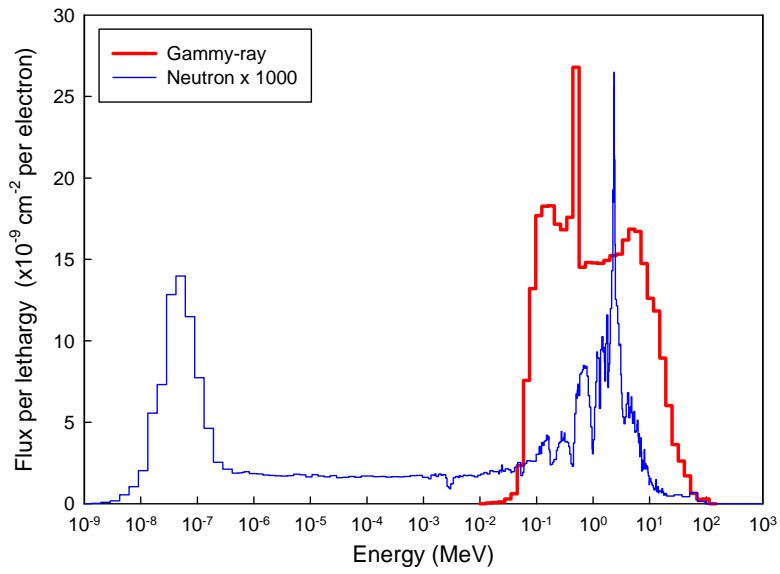

Figure 6. Neutron and gamma-ray energy spectra at the measurement location calculated for electrons lost near the $4^{\text {th }}$ quadruple.

Table 1. Comparison of measured neutron counting rates (counts per minute), neutron and gamma-ray dose rates ( $\left.\mu \mathrm{Sv} \mathrm{h}^{-1}\right)$ with the calculated results. Three beam loss scenarios are considered: 100\% lost at the beam dump (100\%DP), 100\% lost at Q4 (100\%Q4), and a combined scenario with $98 \%$ lost at the beam dump and $2 \%$ lost at Q4 (98\%DP+2\%Q4).

\begin{tabular}{lcccc}
\hline LINAC $(2.25 \mathrm{~W})$ & Meas. & Calc. $(100 \% \mathrm{DP})$ & Calc. $(100 \% \mathrm{Q} 4)$ & Calc. $(98 \% \mathrm{DP}+2 \% \mathrm{Q} 4)$ \\
\hline Bare $(\mathrm{cpm})$ & $386.4 \pm 5.4 \%$ & $1112.4 \pm 18.7 \%$ & $8995.2 \pm 5.4 \%$ & $1270.0 \pm 16.0 \%$ \\
$\mathrm{PE}(\mathrm{cpm})$ & $287.7 \pm 12.75 \%$ & $141.1 \pm 8.2 \%$ & $9123.1 \pm 0.6 \%$ & $320.7 \pm 3.6 \%$ \\
$\mathrm{PE}+\mathrm{Pb}(\mathrm{cpm})$ & $536.2 \pm 4.5 \%$ & $142.8 \pm 8.5 \%$ & $17146.4 \pm 2.3 \%$ & $482.8 \pm 3.0 \%$ \\
Neutron $(\mu \mathrm{Sv} / \mathrm{h})$ & $0.15 \pm 12.75 \%$ & $0.05 \pm 28.1 \%$ & $3.80 \pm 1.9 \%$ & $0.13 \pm 11.3 \%$ \\
Gamma-ray $(\mu \mathrm{Sv} / \mathrm{h})$ & $6.38 \pm 1.7 \%$ & $0.12 \pm 3.4 \%$ & $172.05 \pm 2.4 \%$ & $3.56 \pm 2.3 \%$ \\
\hline
\end{tabular}

rays are between 0.1 and $10 \mathrm{MeV}$ and most neutrons are in thermal range for electrons lost at the beam dump. For electrons lost at Q4, the resultant spectra are somewhat different and have important implications for detector responses. Number of neutrons in $\mathrm{MeV}$ range is roughly comparable to that of thermal neutrons. High-energy neutrons $(\geq 10 \mathrm{MeV})$ are relatively minor in intensity. It is worth noting that there is an obvious high-energy tail $(\geq 10 \mathrm{MeV}$ ) in the gamma-ray spectrum of Figure 6 . Those high-energy gamma rays may induce photonuclear reactions in particular when they interact with high-Z materials. Although the interaction probabilities of photonuclear reactions are probably lower than that of photoatomic reactions by a factor of 100 or more, number of photoneutrons and their contribution to a neutron detector could be important due to copious gamma rays existed in the radiation field.

\subsection{Measurements and data analysis}

Three high-sensitivity neutron detectors (Bare, PE, and $\mathrm{PE}+\mathrm{Pb}$ ) and a high-pressure ionization chamber (HPIC) were used to measure neutron and gamma rays outside the downstream shielding wall during a full-power beam testing. The measured counting rates of three neutron detectors and dose rates for neutrons and gamma rays are listed in Table $\mathbf{1}$. The measured gamma-ray dose rate of $6.38 \mu \mathrm{Sv} \mathrm{h}^{-1}$ is higher than that of neutrons $\left(0.15 \mu \mathrm{Sv} \mathrm{h}^{-1}\right)$ and also higher than what we have expected $\left(0.12 \mu \mathrm{Sv} \mathrm{h}{ }^{-1}\right)$ if all the accelerated electrons were dumped into their final destination. Besides, the measured counting rate of the $\mathrm{PE}+\mathrm{Pb}$ neutron detector is almost twice that of the PE detector, which surprised us since we didn't expect so many high-energy neutrons for the operation of a $150-\mathrm{MeV}$ electron accelerator. In order to examine the details of the radiation field, we have compared the measured results with a series of calculated detector responses, which were obtained by folding the detector response functions with the FLUKA predicted neutron and gamma-ray spectra at the location of measurement resulting from various beam loss scenarios. For example, Table 1 also lists three sets of calculated detector responses for $100 \%$ beam lost at the beam dump, $100 \%$ beam lost at Q4, and a combined scenario of $98 \%$ lost at the beam dump and $2 \%$ lost at Q4, respectively.

Although most of the accelerated electrons are indeed successfully sent into the beam dump, our previous study indicated that a small portion of electrons lost near Q4 play a dominant role in the magnitude of downstream radiation [6]. The assumption of $2 \%$ beam lost at Q4 and $98 \%$ beam lost at the beam dump was the most likely scenario for the LINAC operation during that time. Except for the bare neutron detector, the overall agreement between the estimated and measured detector responses in Table 1 is generally satisfactory considering many uncertainties in calculation assumptions and accelerator operation conditions. A rather large discrepancy between calculation and measurement exists for the response of the bare neutron detector, which is 
mainly sensitive to thermal neutrons. Most of the discrepancy is believed to be related to the insufficient modeling of the surrounding environment in the simulation, such as miscellaneous objects around the accelerator/detector or soil and ground conditions that might affect the scattering and distribution of thermal neutrons in the field measurement. Further studies are necessary to investigate this discrepancy.

Another interesting observation is the significant contribution of photoneutrons to the $\mathrm{PE}+\mathrm{Pb}$ detector. The detector was originally designed to increase the detection efficiency of high-energy neutrons. However, according to the calculated neutron spectra in Figures 5 and 6, neutrons with energies higher than $10 \mathrm{MeV}$ only amount to a minor portion of total neutrons at the measurement location. In contrast, photoneutrons induced by high-energy gamma rays indeed contribute to neutron counts of the $\mathrm{PE}+\mathrm{Pb}$ detector by a large share. Folding the calculated gamma-ray spectrum at the measurement location with the detector response functions in Figure 4, we can estimate the photoneutron contribution to each of the three neutron detectors. As summarized in Table 2, photoneutrons do not have any contribution to the response of the bare detector but account for about 3.7\% of neutron counts registered by the PE detector. The most surprising result is for the $\mathrm{PE}+\mathrm{Pb}$ detector, in which approximately $38 \%$ of neutron counts are coming from photoneutrons. The counting rate ratio of the $\mathrm{PE}+\mathrm{Pb}$ detector to the PE detector could be originally regarded as an indicator of high-energy neutron significance for a specific radiation field. Here it is however related to high-energy gamma rays. The higher the ratio, the more counts registered by the detector are resulting from photoneutrons originated from the detector itself. In this study, the measured ratio is $1.86(536.2 / 287.7)$ while the calculated ratio is 1.51 (482.8/320.7). This number indicates that there are non-negligible high-energy gamma rays in the radiation field and the photoneutron contribution to the detector response should be carefully evaluated. It also suggests that using an extended range neutron detector in an environment with high-energy gamma rays may overestimate the ambient neutron dose rates.

Table 2. Neutron and photoneutron relative contributions to the responses of three neutron detectors.

\begin{tabular}{lccc}
\hline LINAC (2.25W) & Calc. & Neutrons & Photoneutrons \\
\hline Bare (cpm) & 1270.0 & $100 \%$ & $0.0 \%$ \\
PE (cpm) & 320.7 & $96.3 \%$ & $3.7 \%$ \\
PE+Pb (cpm) & 482.8 & $61.6 \%$ & $38.4 \%$ \\
\hline
\end{tabular}

\section{Concluding remarks}

Three high-sensitivity moderated-type neutron detectors were assembled, calibrated, and tested at the TPS LINAC. Detector response functions to neutrons and gamma rays with various energies were calculated by continuous-energy MCNPX calculations. Absolute neutron and gamma-ray spectra at the location of measurement were estimated by FLUKA simulations with a rather detailed geometry modeling of the LINAC room. Total counting rates of the detectors can be estimated by folding the resultant neutron and gamma-ray spectra with corresponding detector response functions. Although most of the accelerated electrons are guided into the beam dump, the study confirms that a small portion of electrons lost near Q4 at the transfer line plays a dominant role in the downstream radiation field. Based on the assumption of $2 \%$ beam lost at Q4 and $98 \%$ at the beam dump, the calculated results of the detector responses are mostly consistent with the measurements showing that the response functions, the characteristics of radiation field, and the estimated beam loss scenario should be reasonable. For neutron measurements outside the downstream shielding wall of the TPS LINAC, high-energy neutrons $(>10 \mathrm{MeV})$ are negligible, but photoneutrons caused by interactions of high-energy gamma rays with the detector could be a significant factor in the total response, especially for an extended range neutron detector. These high-sensitivity neutron detectors and experiences learned from the study are useful and valuable for radiation monitoring around the area and for future TPS commissioning.

\section{Acknowledgements}

The authors would like to thank the LINAC group in NSRRC for their great help in this study. This work was supported by the National Science Council and Atomic Energy Council in Taiwan, under contract no. NSC101-2623-E-007-007-NU.

\section{References}

[1] NSRRC, Taiwan Photon Source (TPS) Design Handbook, National Synchrotron Radiation Research Center, (2009).

[2] D.B. Pelowitz (Ed), MCNPX User's Manual, Version 2.7.0, LA-CP-11-00438, Los Alamos National Laboratory, (2011).

[3] A. Fasso`, A. Ferrari, J. Ranft and P.R. Sala, FLUKA: A Multi-Particle Transport Code, CERN-2005-10, INFN/TC_05/11, SLAC-R-773 (2005).

[4] G. Battistoni, S. Muraro, P.R. Sala, F. Cerutti, A. Ferrari, S. Roesler, A. Fasso and J. Ranft, The FLUKA code: Description and benchmarking, Proc. Hadronic Shower Simulation Workshop 2006, Sep. 6-8, Fermilab, (2006). M. Albrow and R. Raja Eds., AIP Conference Proceeding 896, (2007), pp.31-49.

[5] W.P. Swanson, Radiological Safety Aspects of the Operation of Electron Linear Accelerators, IAEA Technical Reports Series No. 188, International Atomic Energy Agency, (1979).

[6] A.Y. Chen, Y.C. Lin and R.J. Sheu, A study of beam loss pattern and dose distribution around the TPS LINAC during beam commissioning, Prog. in Nucl. Sci. and Technol. (this issue). 